\title{
ADVANCED DETECTION METHODS OF RADIO SIGNALS FROM COSMIC RAYS FOR KASCADE GRANDE AND AUGER
}

\author{
H. GEMMEKE ${ }^{\prime}$, W.D. APEL ${ }^{\wedge}$, F.A. BADEA ${ }^{\wedge}$, L. BÄHREN ${ }^{b}$, K. BEKK $^{\wedge}$, A.
} BERCUCI $^{C}$, M. BERTAINA ${ }^{l}$, P.L. BIERMANN ${ }^{E}$, J. BLÜMER ${ }^{\Lambda ; i}$, , H. BOZDOG ${ }^{A}$, I.M. BRANCUS ${ }^{C}$, M. BRÜGGEMANN ${ }^{G}$, P. BUCHHOLZ $^{G}$, S. BUITINK ${ }^{H}$, H. BUTCHER ${ }^{\prime \prime}$, A. CHIAVASSA ${ }^{\prime \prime}$, K. DAUMILLER $^{A}$, A.G. DE BRUYN ${ }^{\prime \prime}$, C.M. DE VOS ${ }^{\prime}$, F. DI PIERRO ${ }^{\prime \prime}$, P. DOLL ${ }^{A}$, R. ENGEL ${ }^{A}$, H. FALCKE ${ }^{B ; * ; H}$, P.L. GHIA ${ }^{\prime}$, R. GLASSTETTER ${ }^{K}$, C. GRUPEN ${ }^{\prime}$, A. HAUNGS $^{A}$, D. HECK ${ }^{A}$, J.R. HÖRANDEL ${ }^{\prime}$, A. HORNEFFER ${ }^{H: E}$, T. HUEGE ${ }^{A: E}$, K.-H. KAMPERT ${ }^{K}$, G.W. KANT ${ }^{B}$, U. KLEIN $^{L}$,

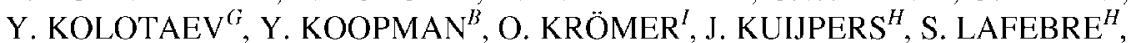
G. MAIER ${ }^{A}$, H.J. MATHES ${ }^{A}$, H.J. MAYER ${ }^{A}$, J. MILKE ${ }^{A}$, B. MITRICA ${ }^{C}$,

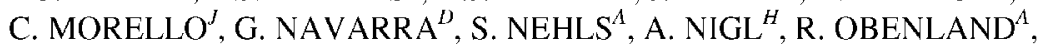

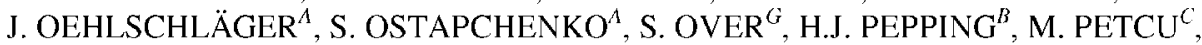
J. PETROVIC ${ }^{H}$, T. PIEROG ${ }^{A}$, S. PLEWNIA ${ }^{A}$, H. REBEL ${ }^{A}$, A. RISSE ${ }^{M}$, M. ROTH $^{I}$, H. SCHIELER ${ }^{A}$, G. SCHOONDERBEEK ${ }^{B}$, O. SIMA ${ }^{C}$, M. STÜMPERT ${ }^{l}$, G. TOMA ${ }^{C}$, G.C. TRINCHERO ${ }^{I}$, H. ULRICH ${ }^{A}$, J. VAN BUREN ${ }^{A}$, W. VAN CAPELLEN ${ }^{B}$, W. WALKOWIAK ${ }^{C}$, A. WEINDL ${ }^{\Lambda}$, S. WIJNHOLDS ${ }^{B}$, J. WOCHELE ${ }^{\Lambda}$, J. ZABIEROWSKI ${ }^{M}$, J.A. ZENSUS ${ }^{E}$, D. ZIMMERMANN ${ }^{(i}$

${ }^{\wedge}$ Institut für Kernphysik, Forschungszentrum Karlsruhe, Germany

${ }^{13}$ ASTRON Dwingeloo, The Netherlands

${ }^{C}$ National Inst. of Physics and Nuclear Engineering, Bucharest, Romania

${ }^{I}$ Dept. of General Physics, Universitá degli Studi di Torino, Italy

${ }^{E}$ Max-Planck-Institut für Radioastronomie, Bonn, Germany

${ }^{F}$ Institut für Experimentelle Kernphysik, Universität Karlsruhe, Germany,

${ }^{\prime}$ Fachbereich Physik, Universität Siegen, Germany

${ }^{H}$ Dept. of Astrophysics, Radboud University Nijmegen, The Netherlands

${ }^{I} I P E$, Forschungszentrum Karlsruhe, Germany

${ }^{J}$ Institute for Interplanetary Space Physics INAF, Torino, Italy

${ }^{K}$ Fachbereich Physik, Universität Wuppertal, Germany

${ }^{L}$ Radioastronomisches Institut, Universität Bonn, Germany

${ }^{M}$ Soltan Institute for Nuclear Studies, Lodz, Poland

The LOPES experiment (LOfar Prototype Station) has been built at the KASCADEGrande experiment in order to test the LOFAR (LOw Frequency ARray) technology and demonstrate its capability for radio measurements in Extensive Air Showers (EAS). After the first positive results in the framework of the KASCADE-Grande experiment we developed the next generation of antennas, electronics, and trigger. The main new features are easy calibration of antennas with precise detection of polarization, and good capability of self-triggering. The results from this new design are under test in Karlsruhe. Furthermore the background situation was measured and analyzed. 


\section{Introduction}

Geo-synchrotron radio emission by high-energy cosmic ray air showers is caused by the deflections of electrons and positrons in the earth's magnetic field. As in synchrotron radiation this radiation is narrowly beamed into the forward direction of the shower. The maximum radio emission is expected in the low frequency range $(10 \mathrm{MHz}$ to $200 \mathrm{MHz}$ ) where the atmospheric attenuation of radio signals below $10 \mathrm{~km}$ height can be neglected and a bolometric measure of the electromagnetic energy content of the shower is achieved. To evaluate this method a phased array of dipole antennas (LOPES) with digital RF processing has been built in co-location with the KASCADE-Grande air shower experiment to ensure the interpretation of the measured data.

The antenna array of LOPES consists of thirty short "inverted V" dipoles operating in the $40 \mathrm{MHz}$ to $80 \mathrm{MHz}$ range. The data readout is triggered by KASCADE-Grande events. The first results ${ }^{1}$ verify the coherent radio emission predicted by the geo-synchrotron effect.

Parallel with these measurements we started LOPES*, an optimization of the antenna design and its electronics for the application in large cosmic ray experiments like the Pierre Auger observatory. LOPES* (LOPES ${ }^{\text {STAR }}$ ) is the acronym for a Self Triggered Array of Radio detectors for LOPES. The main focus of LOPES* is the efficient suppression of man-made radio frequency interference (RFI), an antenna design which can be simply calibrated, with low power consumption for operating with a solar and battery supply and with the feature of self-triggering. LOPES* works in the same frequency range as LOPES.

\section{Choice of Antenna for LOPES*}

For the choice of antenna we considered and tested 3 types: the logarithmic periodic conical helix used in the CODALEMA ${ }^{2}$ experiment, the inverted $\mathrm{V}$-type antenna of LOPES 3 and the crossed logarithmic-periodic dipole antenna ${ }^{4}$ (crossed LPDA). The conical helix antenna was discarded because of its large crosstalk between both polarization directions of about $-6 \mathrm{~dB}$, if both antennas are mounted

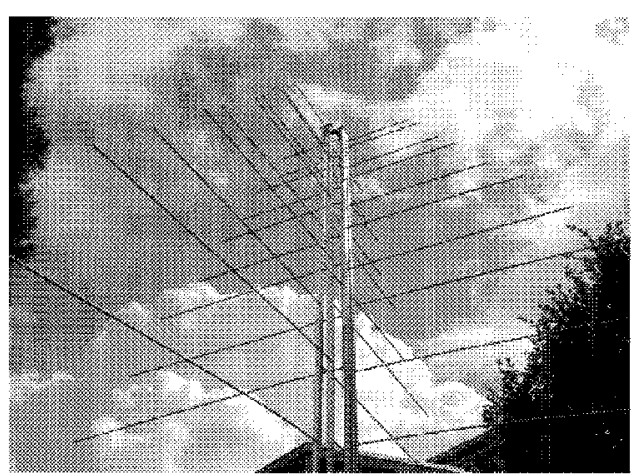

Figure 1. Crossed logarithmic-periodic dipole antennas of LOPES*. 
on top of each other on the same pole. Furthermore it is expensive to build a heightened version protected against environmental effects at ground.

The V-type antenna is simple to construct and inexpensive. But it has a frequency dependent sensitivity and impedance with a high capacitive component, which may be partly compensated by the preamplifier. That makes calibration and data analysis difficult. The horizontal attenuation is very weak and increases the sensitivity to man-made RFI. The missing backward attenuation must be compensated by a defined ground layer otherwise the calibration of the antenna changes. The crossed LPDA in Fig.1 does not have any of these disadvantages, see Table 1 , is in the overall costs for production and installation not considerably more expensive than the V-type antenna, but cheaper than the conical helix.

Table 1. Measured characteristics of the crossed LPD antennas

\begin{tabular}{ll}
\hline Characteristic & Measured values \\
\hline Bandwidth: & $35 \ldots 90 \mathrm{MHz}$ \\
Gain: & $5.5 \mathrm{dBi}^{*}$ \\
Impedance: & Real, $50 \mathrm{Ohm}$ \\
Return loss: & $-12 \mathrm{~dB}$ \\
Beam width [-3dB]: & $\pm 50^{\circ}$ (E-Plane), $\pm 70^{\circ}$ (H-Plane) \\
$\pm 90^{\circ}$-attenuation: & $20 \mathrm{~dB}$ (E-Plane), $6 \mathrm{~dB}$ (H-Plane) \\
Backward attenuation: & $20 \mathrm{~dB}$ \\
Polarisation isolation: & $>20 \mathrm{~dB}$ \\
Size (without pole): & $4 \times 4 \times 3 \mathrm{~m}^{3}$ \\
Weight (without pole): & $15 \mathrm{~kg}$ \\
\hline
\end{tabular}

The crossed LPDA can be built with any user-defined bandwidth. The directivity has a strong forward characteristic of $5.5 \mathrm{dBi}^{*}$, a high backward attenuation and a high rejection in the horizontal direction, suppressing manmade RFI and interaction with ground and buildings efficiently. Using a crossed assembly all polarisations may be received and distinguished, therefore we choose the crossed LPDA.

\section{Low power front-end electronics and measured radio background}

The RF is amplified close to the antenna with a Low Noise Amplifier (LNA) with $1.8 \mathrm{~dB}$ noise-figure and a power consumption of $22 \mathrm{~mW}$ per channel. The remaining part of the analog frontend has a power consumption of $65 \mathrm{~mW}$ per channel. Both preamplifier and frontend fulfil our solar power budget of $100 \mathrm{~mW}$ per channel. The anti-aliasing band-pass filter is of $32^{\text {nd }}$ order and uses

\footnotetext{
"The unit $\mathrm{dBi}$ is the gain of the antenna relative to an isotropic radiator.
} 
nearly the whole $2^{\text {nd }}$ Nyquist domain at $80 \mathrm{MHz}$ sampling rate from $41 \mathrm{MHz}$ to $79 \mathrm{MHz}$ with only $3 \mathrm{~dB}$ passband ripple and $10 \mathrm{~dB} / \mathrm{MHz}$ slope at the band limits. To reject all high-level out-of-band components (Fig. 2) the stopband attenuation is $110 \mathrm{~dB}$ at shortwave, $90 \mathrm{~dB}$ at the FM band, and $80 \mathrm{~dB}$ at the VHF III band.

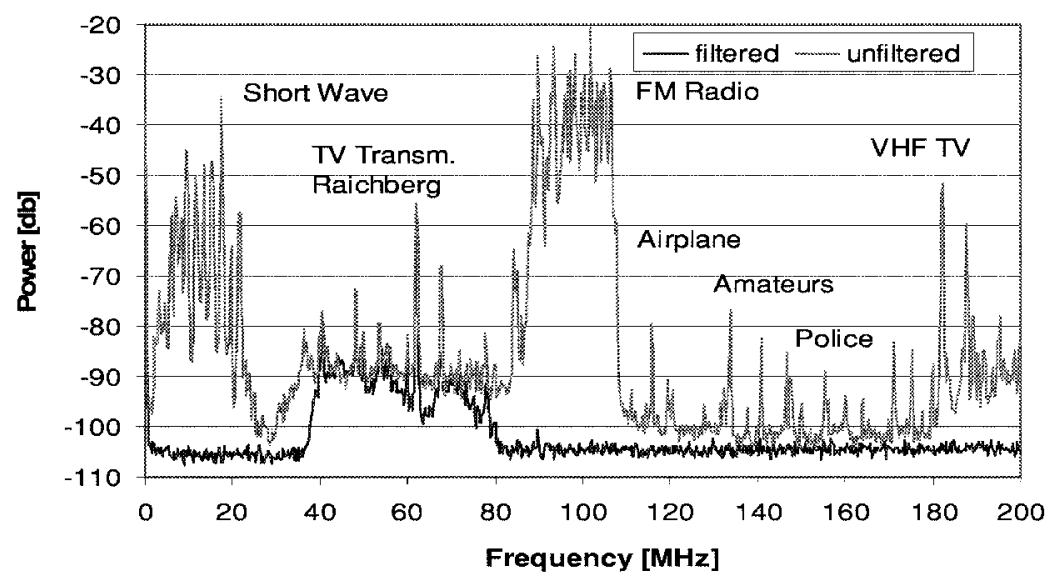

Figure 2. Measured radio background at FZ Karlsruhe and the effect of our filter.

The measured out-of-band background at Forschungszentrum Karlsruhe will be similar elsewhere and its suppression requires such strong filters. A commercial VME DAQ ${ }^{5}$ system performs a 12 bit AD-conversion with $80 \mathrm{MHz}$ sampling rate and stores the data in two ring buffers.

\section{Trigger}

The basic antenna array setup for self-triggering consists of three crossed LPDAs on the edges of an equilateral triangle. The triangle setup at Forschungszentrum Karlsruhe has a side length of $65 \mathrm{~m}$ (Fig. 3). The trigger is activated only, if all three antennas produce signals within the same 190 ns time slot given by the height of the triangle divided by the speed of light. Thereby the trigger hardware is able to distinguish most of the signals from the horizon, which are normally man made, from signals with higher elevation, which may be excited by air showers. So the trigger not only demands signals at all antennas above the thresholds, but also discriminates the elevation angle (horizontal or elevated). RFI-sources inside the trigger-triangle have to be avoided, because they can't be discriminated from real shower events by their timing. 
The envelope signal (Fig. 4) is given by the RF power and produces short pulses at air showers while many man made RFI cause only slow amplitude changes, which may be easily removed by high-pass filtering. At present the first self triggered measurements have been started to investigate the dependence of trigger rates on the coincidence parameters. All events are GPS time stamped to find coincidences with KASCADE-Grande triggers.

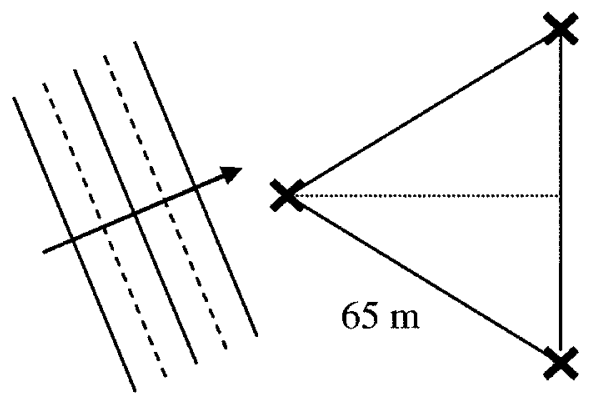

Figure 3. Triangular antenna geometry and a horizontal interference source.

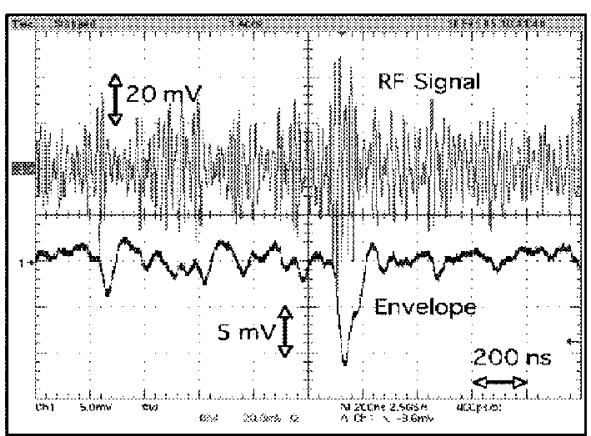

Figure 4. Triggering with envelope signals.

\section{Conclusion and future steps}

So far a test array of three antennas is installed in Karlsruhe and data are recorded. The expected background rejection and self-trigger features could be demonstrated. The installation is now used to measure the obtainable time resolution. The installation of several triangular setups in Karlsruhe is in preparation. A similar installation is planned on the site of the southern Pierre Auger Observatory, especially to optimize the geometry for such an array to measure energies above $10^{19} \mathrm{eV}$ under realistic conditions.

\section{References}

1. H. Falcke et al., Nature 435, 313 (2005)

2. D. Ardouin et al., CODALEMA, astro-ph/0504297, submitted to NIM A

3. A. Horneffer et al., Proc. SPIE 5500-21, (2004)

4. Rothamme1, 12. Auflage, 2001, S. $635-646$

5. SIS3300, SIS GmbH, 22399 Hamburg, Germany, http://www.struck.de 